\title{
Energy Efficient Watermarking on Mobile Devices Using Proxy-Based Partitioning
}

\author{
Arun Kejariwal, Student Member, IEEE, Sumit Gupta, Member, IEEE, Alexandru Nicolau, Member, IEEE, \\ Nikil D. Dutt, Senior Member, IEEE, and Rajesh Gupta, Fellow, IEEE
}

\begin{abstract}
Digital watermarking embeds an imperceptible signature or watermark in a digital file containing audio, image, text, or video data. The watermark can be used to authenticate the data file and for tamper detection. It is particularly valuable in the use and exchange of digital media, such as audio and video, on emerging handheld devices. However, watermarking is computationally expensive and adds to the drain of the available energy in handheld devices. In this paper, we first analyze the energy profile of various watermarking algorithms. We also study the impact of security and image quality on energy consumption. Second, we present an approach in which we partition the watermarking embedding and extraction algorithms and migrate some tasks to a proxy server. This leads to a lower energy consumption on the handheld without compromising the security of the watermarking process. Experimental results show that executing the watermarking tasks that are partitioned between the proxy and the handheld devices, reduces the total energy consumed by $80 \%$, and improves performance by two orders of magnitude compared to running the application on only the handheld device.
\end{abstract}

Index Terms-Energy consumption, mobile computing, partitioning, watermarking.

\section{INTRODUCTION}

$\mathbf{T}$ THE INCREASING computational capability and availability of broadband in emerging handheld devices have made them true endpoints of the internet. They enable users to download and exchange a wide variety of media such as e-books, images, etc. However, practical use of these devices requires effective protection of Internet Protocol (IP) rights associated with such media.

Digital watermarking [1]-[4] has been proposed as a technique for protecting intellectual property of digital data. It is the process of embedding a signature/watermark into a digital media file so that it is hidden from view, but can be extracted on demand to verify the authenticity of the media file. The watermark can be a binary data, a logo, or a seed value to a pseudorandom number generator to produce a sequence of numbers with a certain distribution (e.g., Gaussian or uniform).

Manuscript received March 21, 2005; revised February 18, 2006 and March 17,2006 . This is an expanded version of the paper that appeared at the Design Automation Conference in June, 2004. This work was supported in part by the National Science Foundation (NSF) under Grant ACI-0204028.

A. Kejariwal, S. Gupta, A. Nicolau, and N. D. Dutt are with the Department of Computer Science, University of California at Irvine, Irvine, CA 92697 USA (e-mail: arun_kejariwal@ieee.org).

R. Gupta was with the Department of Computer Science, University of California at Irvine, Irvine, CA 92697 USA. He is now with the Department of Computer Science and Engineering, University of California at San Diego, La Jolla, CA 92093 USA.

Digital Object Identifier 10.1109/TVLSI.2006.878218
Several watermarking-based techniques have been proposed recently to address these concerns, such as Sony's Signet Screener technology ${ }^{1}$ for video and Light Weight DRM (Digital Rights Management) from Germany's Fraunhofer Institute. Similarly, watermarking can be used to combat fraudulent use of wireless voice communications, authenticating the identity of cell phones and transmission stations, and securing the delivery of music and other audio content. ${ }^{2}$ Bauerle et al. suggest watermarking images taken by the inbuilt camera in a mobile device (cell phone, PDA) or the global positioning satellite (GPS) coordinates of the user before sending it to the server over a nonsecure wireless channel. Products like Digimarc ImageBridge, ${ }^{2}$ Verance's watermarking tools ${ }^{3}$ for Hitachi's 32-bit RISC processor SH-Mobile (SuperH Mobile Application Processor) allow communicating copyrights in digital images and tracking those images on the Internet. Similarly, RealOne Player ${ }^{4}$ for mobile devices like the Nokia 9210/9290 and the Compaq iPAQ Pocket PC, provides support to play watermarked streaming RealAudio and RealVideo files live and on-demand. Further, security concerns in mobile e-commerce have been discussed in [5]. Watermarking bears a large potential in securing such applications, for example, e-fax for owner verification, customer authentication in service delivery, and customer support.

Since mobile devices such as cell phones, PDAs, and music players have become ubiquitous, the use of watermarking or some similar technique for the protection of digital media rights on these devices is inevitable [6]. However, watermarking algorithms are designed for maximum security with little or no consideration for other system constraints such as computational complexity and energy availability. Handheld devices such as PDAs and cell phones have a limited battery life that is directly affected by the amount of computational burden placed by the application. Digital watermarking tasks place an additional burden on the available energy in these devices.

In this paper, we first analyze the energy profile of various watermarking algorithms. We also study the impact of security and image quality on energy consumption. Second, we propose a task partitioning scheme for wavelet-based image watermarking algorithms in which computationally expensive portions of the watermarking are "offloaded" to a proxy server. The proxy server acts as an agent between the content server and the handheld device and is used for various other tasks such

\footnotetext{
${ }^{1}$ http://www.signetscreener.com/

${ }^{2} \mathrm{http}: / / \mathrm{www}$.digimarc.com

${ }^{3} \mathrm{http}: / / \mathrm{www} . v e r a n c e . c o m / n e w s /$ releases/09-17-02.html

${ }^{4} \mathrm{http}: / /$ www.real.com
} 


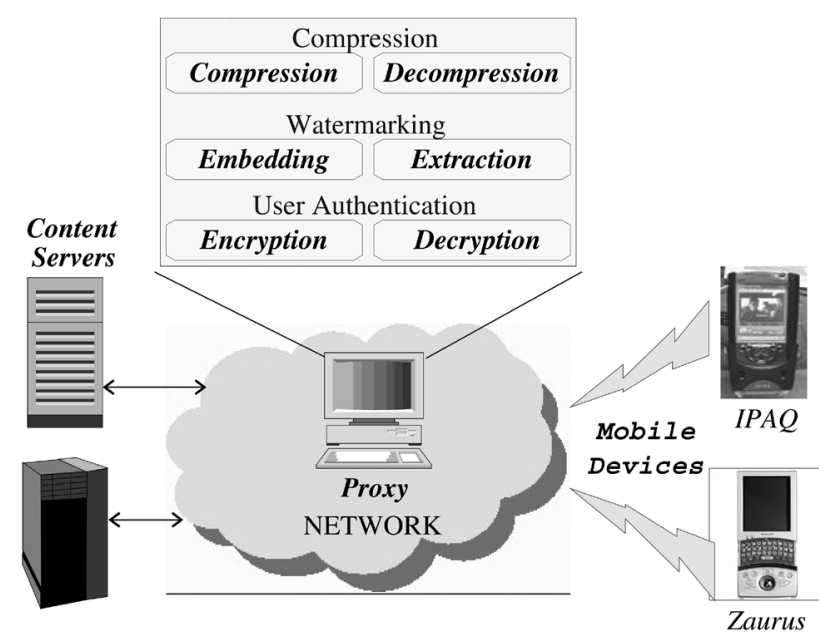

Fig. 1. Architecture of target system.

as data transcoding, load management, etc. We show how our partitioning scheme can be used to reduce energy consumption associated with watermarking on the handheld without compromising the security of the watermarking process.

The rest of the paper is organized as follows. In Section II, we present related work. Next, we present the architecture of our target system. In Section IV, we present an overview of the watermarking process. Next, we discuss wavelet-based watermarking. Our proxy-based partitioning approaches for watermarking on the handhelds in case of both trusted and nontrusted proxy, have been described in Section VI. In Section VII, we present our experimental setup. Section VIII discusses the energy analysis of various watermarking algorithms. The impact of security and image quality on energy consumption during the watermarking process have been studied in Section IX. Results of the study of the performance-security tradeoffs associated with our partitioning approaches are presented in Section X. Finally, we conclude our work in Section XI.

\section{RELATED WORK}

This work is based upon ongoing advances in digital watermarking and proxy-based middleware services. The term watermark traditionally refers to an almost imperceptible imprint on paper that marks the authenticity of the document. Digital watermarking extends this idea to digital content [1]-[4]. Watermarking, like steganography, seeks to hide information inside another object. Therefore, it should be resilient to intentional or unintentional manipulations and resistant to watermark attacks [2], [7] (except in the case of fragile watermarks).

The use of proxies as agents that can connect to a range of heterogeneous clients is a well-established practice [8], [9]. Several approaches have been proposed for securing the connection between the proxy and mobile devices that they serve [10], [11]. Also, moving computationally expensive tasks to proxies has been discussed in the past [12] and recently, is used in the context of establishing secure connections [9], [13]. However, in most of these works, task mapping is driven solely by performance, ignoring power constraints that are critical for handheld mobile devices. Recently, Potlapally et al. [14] analyzed the energy requirements of a wide range of cryptographic al-

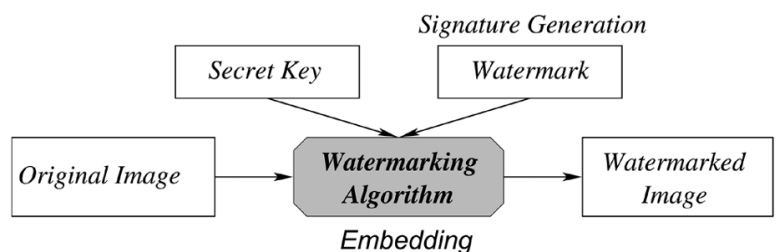

(a)

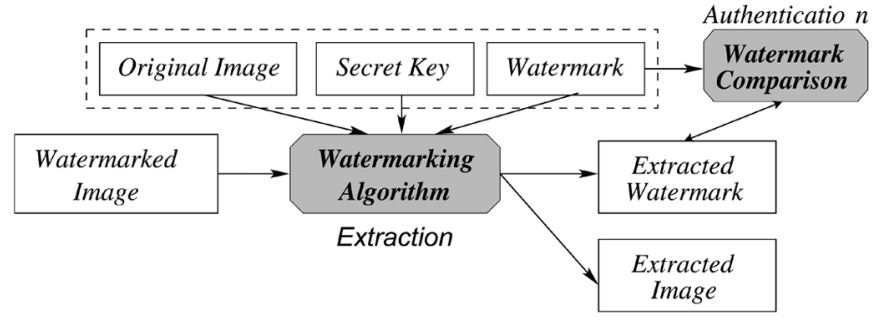

(b)

Fig. 2. Watermarking process: (a) watermark generation and embedding and (b) watermark extraction and authentication.

gorithms used as building blocks in security protocols such as secure sockets layer (SSL). Although several techniqes have been proposed for remote task execution for power management [15]-[17], these do not account for the application security during the partitioning process. Furthermore, these techniques are not generic in nature; thus, they are inapplicable in our context.

\section{SYSTEM ARCHITECTURE}

Fig. 1 shows our implementation of a watermarking system in which multimedia content is streamed to a handheld device via a proxy server. This system consists of three components: mobile devices, proxy servers, and content servers. A mobile or handheld device refers to any type of networked resource; it could be handheld (PDA), a gaming device, or a wireless security camera. Content servers store multimedia and database content and stream data (images) to a client as per request. All communication between the mobile devices and the servers are relayed through the proxy servers. Proxy servers are powerful servers that can, among other things, compress/decompress images, transcode video in real-time, access/provide directory services, and provide services based on a rule base for specific devices. Thus, mobile devices actually negotiate with proxy servers for security, quality of service, and content delivery. The proxy servers, in turn, request the content servers for the image/video/data stream as per user requirements. The transmission of multimedia data from the content server to the proxy is assumed to be secure. Note that mobile devices may also create and send data through proxy servers to other mobile devices in the network.

In Section VI, we discuss techniques that exploit the capabilities of proxy servers to dynamically offload watermarking tasks from handheld devices under constraints on the bandwidth available to the client devices.

\section{WATERMARKING}

Fig. 2 shows the general process of watermarking image data, where the original image (host image) is modified using a signature to create the watermarked image. In this process, some 


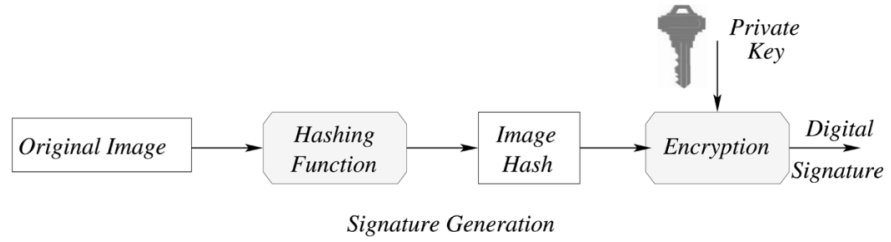

Fig. 3. Digital signature generation.

error or distortion is introduced. To ensure transparency of the embedded data, the amount of image distortion due to the watermark embedding process has to be small. There are three basic tasks in the watermarking process with respect to an image as shown in Fig. 2(a) and (b). These are as follows.

- Embedding: A watermark is embedded either in the spatial domain (by modifying the relationship of a pixel with its neighboring pixels) or in the frequency domain [by modifying the discrete cosine transform/discrete wavelet transform (DCT/DWT) coefficients]. Embedding a watermark into an image may use a secret key to determine the way in which the watermark is embedded into the image [2].

- Detection and Extraction: Refers to detecting whether an image has a watermark and extracting the full watermark from the image. The watermark is extracted from the image by applying a process that is almost the inverse of the embedding process.

- Authentication: Refers to comparing the extracted watermark with the original watermark. In general, a threshold is defined for the comparison. If the differences (if any) are more than the threshold, then the image is declared as tampered.

Next, we discuss the process of generating a watermark or signature and two applications for watermarking.

\section{A. Watermark/Signature Generation}

Watermarks (or digital signatures) are used to detect unauthorized modifications of data and for ownership authentication. In addition, the recipient of signed data can use a digital signature in proving to a third party that the signature was, in fact, generated by the signer of the data. This is known as nonrepudiation (or fingerprinting) since the signer of data cannot, at a later time, repudiate the signature.

A watermark (digital signature) is created in two steps, as shown in Fig. 3.

1) A fingerprint of the image is created using a one-way hashing function.

2) The hash value is encrypted with the private key of a private-public key pair. Thus, forging a signature without the knowledge of the private key becomes infeasible.

\section{B. Watermarking Techniques for Image and Video}

Watermarking techniques for images and video differ in that watermarking in video streams takes advantage of the temporal relation between frames to embed watermarks. This is explained in Section IV-B-I and IV-B-II.
1) Image Watermarking: A simple approach for embedding data into images is to set the least significant bit (LSB) of some pixels to zero [18]. Data is then embedded into the image by assigning 1's to the LSBs in a specific manner (known only to the owner). This method satisfies the perceptual transparency property, since only the least significant bit of an 8-bit value is altered. ${ }^{5}$

In DCT-based watermarking, the original image is divided into $8 \times 8$ blocks of pixels, and the two-dimensional (2-D) DCT is applied independently to each block. The watermark is then embedded into the image by modifying the relationship of the neighboring blocks of the DCT coefficients that are in the middle-frequency range in the original image [19].

Each red-green-blue (RGB) component in color images may have a different resolution. Wavelet-based watermarking techniques exploit this by creating a multiresolution representation in which each RGB component is represented in a different frequency band. This, however, leads to computationally expensive searches for coefficients across sub-bands [20] while embedding and extracting the watermark. The watermark is adaptively weighted into different sub-bands to achieve robustness as well as imperceptibility.

2) Video Watermarking: The spatial and frequency domain watermarking techniques used in still images, are extended to the temporal domain for video streams. In this, one can take advantage of the fact that in MPEG video streams the (predicted) frames and the (bi-directional) frames are derived from reference (intermediate) frames using motion estimation [21]. In this paper, we present energy analysis and partitioning schemes for image watermarking algorithms only.

\section{WAVELET-BASED WATERMARKING}

Wavelet-based watermarking is one of the most popular approaches due to its robustness against malicious attacks [22], [23]. Wavelet-based image watermark embedding consists of three phases: 1) watermark preprocessing; 2) image preprocessing; and 3) watermark embedding, as shown in Fig. 4. First, each bit in each pixel of both the image and the watermark is assigned to a bit plane. There are 8 bit planes corresponding to the gray-level resolution of the image/watermark. Then DWT [24] coefficients are obtained for each bit plane by carrying out DWT on a plane-by-plane basis. The DWT coefficients of the watermark are encrypted using a public key. ${ }^{6}$ The watermark embedding algorithm then uses the coefficients of the original image and those of the encrypted watermark to generate the watermarked image.

A similar reverse process is used for watermark extraction and authentication. First, the encrypted coefficients of the image and the watermark are extracted from the image. Then a secret private key is used to decrypt the coefficients of the watermark and an inverse DWT is applied and so on, till the original watermark is obtained.

\footnotetext{
${ }^{5}$ For simplicity, we assumed an 8-bit representation of a pixel value; the above discussion can be easily extended for other representations, such as 32-bit.

${ }^{6}$ The public and private keys correspond to the popular asymmetric encryption technique [25].
} 


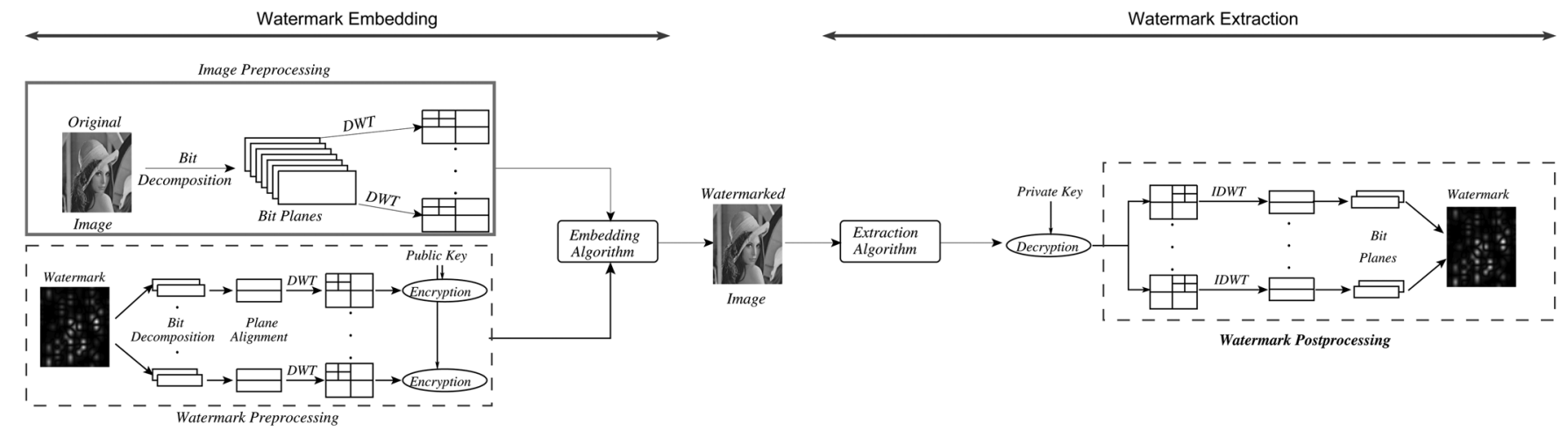

Fig. 4. Image watermark embedding and extraction process (public key used for encryption, whereas private key is used for decryption).

\section{A. DWT}

DWT [24] uses filters with different cutoff frequencies to analyze a signal (image/video, in our case) at different resolutions. ${ }^{7}$ The signal is passed through a series of high-pass filters, also known as wavelet functions, to analyze the high frequencies and it is passed through a series of low-pass filters, also known as scaling functions, to analyze the low frequencies.

After filtering, half of the samples can be eliminated according to the Nyquist rule. Since the signal now has a highest frequency of $p / 2$ radians instead of $p$, the signal can, therefore, be sub-sampled by 2 , simply by discarding every other sample. This constitutes one level of decomposition. Thus, decomposition halves the time resolution (half the number of samples) and doubles the frequency resolution (half the span in the frequency band). The above procedure, also known as the sub-band coding, is repeated for further decomposition in order to make a multiresolution analysis. The number of decompositions levels are predefined during this process.

\section{Partitioning Watermarking Tasks Across the NETWORK}

In this section, we present our approach for partitioning watermarking between the proxy server and the client device. There is a tradeoff between the degree of security available on the mobile devices versus its energy consumption with each mapping. We present two partitioning schemes-the first gives priority to reduction of energy consumption, Section VI-A, while the other gives priority to security of the watermarking procedure, Section VI-B. Note that in the context of our system architecture (shown earlier in Fig. 1), the mobile devices may extract watermarks from received image data or may embed watermarks in images that they send out.

\section{A. Mapping All Watermarking Tasks to the Proxy}

In an attempt to maximize battery life, we "offload" all the image and watermark preprocessing and embedding tasks to the proxy. The only extra communication required is transmitting the watermark and the secret key once from the handheld to the proxy. Whether the handheld is transmitting or receiving,

\footnotetext{
${ }^{7}$ Resolution is used as a measure of the amount of detailed information contained in the image.
}

the data is streamed via the proxy anyway, so the proxy can do watermark embedding, extraction, and/or authentication.

This watermark process migration is applicable in office environments where a trusted proxy can act as an "agent" or representative for the mobile device and can take care of authentication and quality of service negotiation with the content server. In such a scenario, the handheld and the proxy may be connected using a secure SSL connection that prevents watermark interception and tampering.

\section{B. Security-Driven Partitioning}

A more secure partitioning scheme for both watermark embedding and extraction requires some participation from the device in the watermarking process.

During watermark embedding, we migrate the following tasks to the proxy: bit decomposition, coefficient calculation using DWT, and watermark coefficient encryption using the public key. So, the handheld first sends the image and the watermark to the proxy. The proxy processes them and sends the image and watermark coefficients back to the handheld. The handheld then embeds the watermark coefficients into the image using a unique coefficient relationship to generate the watermarked image. This is a secure approach as the proxy does not know the coefficient relationship used to embed the watermark coefficients in the image.

During watermark extraction, the handheld extracts the image and watermark coefficients from the watermarked (received) image and uses its private secure key to decrypt the image and watermark coefficients. The handheld sends the image coefficients to the proxy for processing, such as carrying out inverse DWT; on the other hand, it processes the coefficients of the watermark itself to generate the watermark. Then it authenticates the watermark against the original watermark. The fact that the watermark is not sent to the proxy makes this scheme secure against any potential malacious attack by the proxy. We discuss the communication overhead of this partitioning scheme in the results (see Section X-C). Fig. 5 shows the block diagram of security-driven partitioning of the image watermarking scheme shown in Fig. 4.

\section{EXPERIMENTAL SETUP}

Our experimental setup is shown in Fig. 6. All our measurements were made using a Sharp Zaurus PDA with an Intel 


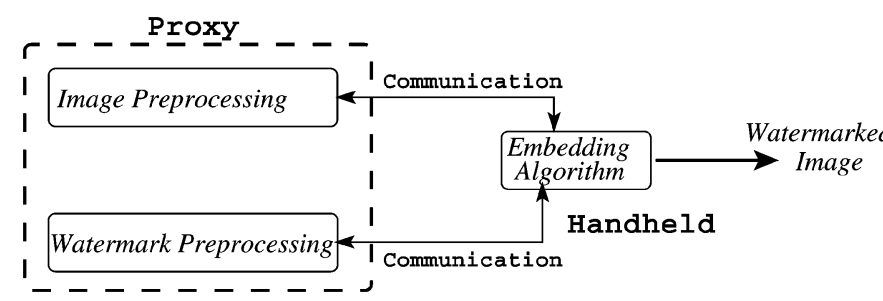

Fig. 5. Partitioned image watermark embedding and extraction process.

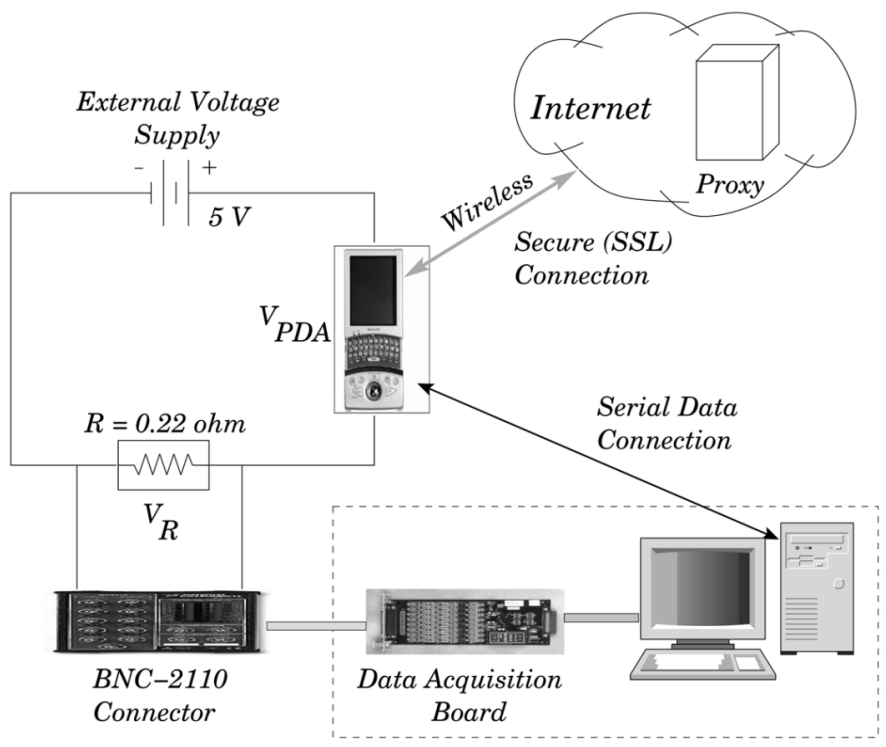

Fig. 6. Experimental setup.

Power Measurement System

400-MHz XScale processor with a 64-MB ROM and 32-MB SDRAM. We used a Belkin 802.11b compact flash network card on the Zaurus for communication. We removed the internal battery from the Zaurus and placed a resistor in series with the power supply, as shown in Fig. 6. We used a National Instruments PCI-6040E data acquisition (DAQ) board to sample the voltage drop across the resistor (to calculate current) at 1000 samples/s. The DAQ has a resolution of 16 bits. We calculated the instantaneous power consumption corresponding to each sample and the total energy using the following equations:

$$
\begin{aligned}
\mathcal{P}_{\text {Inst }} & =\frac{V_{R}}{R} \times V_{\mathrm{PDA}} \\
E & =\sum \mathcal{P}_{\text {Inst }} \times T
\end{aligned}
$$

where $V_{R}$ is the instantaneous voltage drop across the resistor (in volts) with resistance $R \omega$ and $V_{\mathrm{PDA}}$ is the voltage across the Zaurus PDA (or the supply voltage), and $T$ is the sampling period $(T=1 / 1000)$. Energy $E$ is the sum of all the instantaneous power samples for the duration of the execution of the application multiplied by the sampling period $T$. We calculate average power as the ratio of total energy over total execution time. All the energy/power measurements reported in this section are with respect to (w.r.t.) an idle system. In other words, we first determined the power consumption of an idle system. The measurement was made using the setup shown in Fig. 6, while making sure that the PDA is not powered down. This number was subtracted from the energy/power consumption numbers obtained by executing the watermark embedding and extraction algorithms on the PDA. This masks the spurious systems effects due to background/OS tasks, interrupts, network activity, and other processes. Further, the performance and power reported in the rest of this section correspond to an average of 10 runs.

To characterize the energy/power consumption of watermarking on handheld devices, we performed our experiments on 11 image watermarking algorithms discussed in [36] and available from. ${ }^{8}$ The source code was cross-compiled using arm-gcc $c^{9}$ for Zaurus. In Table I, we list a brief description of each of these watermarking algorithms. We also measured the execution time of the watermarking algorithms on a proxy server, an Intel Celeron 1.70-GHz Linux PC machine, connected to the PDA over a 802.11 wireless network.

\section{ENERGY/POWER CONSUMPTION ANALYSIS OF WATERMARKING ON HANDHELD DEVICES}

\section{A. Watermark Embedding}

Table II lists the energy usage, average power (energy/execution time), and execution time for watermark embedding by the various watermarking algorithms when they are executed on the handheld.

From Table II, we note that Bruyndonckx's [26] and Koch's [31] algorithms are the most energy and performance efficient. This can be attributed to their low security levels as compared to other algorithms. For example, Bruyndonckx [26] embeds most of the signature bits in a few blocks in the image, thus, enabling quick, low computation searches. This speeds up the embedding process and leads to low-energy consumption. However, such localized embedding strategies are highly susceptible to watermark attacks [37]. Similarly, Koch [31] embeds signature bits by modifying coefficient ${ }^{10}$ relationships using mean square error to minimize error. As compared to other frequency domain watermark embedding techniques, Koch's algorithm offers a very low-security level owing to the simple embedding criteria. ${ }^{11}$ In contrast, Xie [34] and Zhu [35] employ wavelet-based watermarking techniques; such sub-band level embedding techniques are more secure than other techniques. However, calculating wavelet and inverse-wavelet transforms is computationally expensive and, thus, also power hungry. The large variation in the power consumption of the different algorithms can be in part attributed to the difference in the type of instructions executed in

\footnotetext{
${ }^{8} \mathrm{http} / / / \mathrm{www} . \operatorname{cosy} \cdot$ sbg.ac.at/ pmeerw/Watermarking/source/

${ }^{9} \mathrm{http}: / /$ www.ossh.com/zaurus/mirrors/docs.zaurus.com/linux_compiler_setup_howto.shtml

${ }^{10}$ The coefficients are obtained by carrying out an $8 \times 8$ DCT transform on the input image.

${ }^{11} \mathrm{By}$ a low level of security of an algorithm, we refer to its low computational complexity.
} 
TABLE I

CHARACTERISTICS OF THE WATERMARKING Algorithms

\begin{tabular}{|c|c|l|}
\hline Algorithm & Domain & \multicolumn{1}{|c|}{ Description } \\
\hline \hline Bruyndonckx [31] & Spatial & Embeds a watermark in the blocks based on their luminance gradients \\
\hline Corvi [32] & DWT & Embeds the scaled watermark bits into the image \\
\hline Cox [33] & DCT & Embeds the watermark bits into perceptually most signi ca nt components of the image \\
\hline Dugad [34] & DWT & Embeds a watermark of $n$ normally distributed values into the co-e $\Delta$ cients of a sub-band \\
\hline Fridrich [35] & DCT & Embeds a watermark in the lower and middle frequency coe $\Delta$ cients of the normalized image \\
\hline Kim [36] & DWT & Embeds a sequence of watermarks into sub-bands in each decomposition level \\
\hline Koch [37] & DCT & Embeds the watermark bits by modifying the coe $\Delta$ cient relationship \\
\hline Wang [38] & DWT & Embeds a watermark into selected sub-bands determined by a threshold \\
\hline Xia [39] & DWT & Embeds a watermark into the wavelet transformed image along di@erent directions \\
\hline Xie [40] & DWT & Employs a 3-pixel window to embed a watermark \\
\hline Zhu [41] & DWT & Embeds a watermark into a sub-band of a decomposition containing the largest coe $\Delta$ cient \\
\hline
\end{tabular}

DCT $=$ Discrete Cosine Transform, DWT $=$ Discrete Wavelet Transform

TABLE II

WATERMARK EMBEDDING: ENERGY, POWER, AND EXECUTION TIME ANALYSIS

\begin{tabular}{c|c|c|c}
\hline Algorithm & $\begin{array}{c}\text { Energy } \\
(\mathrm{J})\end{array}$ & $\begin{array}{c}\text { Avg. Power } \\
(\mathrm{W}=\mathrm{J} / \mathrm{s})\end{array}$ & Exec. time (s) \\
\hline \hline Bruyndonckx & 1.47 & 0.11 & 13.46 \\
\hline Corvi & 83.2 & 0.61 & 136.15 \\
\hline Cox & 126 & 1.1 & 115.23 \\
\hline Dugad & 68.7 & 0.50 & 136.64 \\
\hline Fridrich & 196 & 1.15 & 171.0 \\
\hline Kim & 73.5 & 0.52 & 140.81 \\
\hline Koch & 2.19 & 0.17 & 12.64 \\
\hline Wang & 85.8 & 0.61 & 140.2 \\
\hline Xia & 90 & 0.67 & 133.82 \\
\hline Xie & 154.8 & 1.05 & 147.07 \\
\hline Zhu & 163.3 & 1.14 & 143.74 \\
\hline
\end{tabular}

each case. The instruction sequence executed is largely dependent on algorithmic properties (which enable certain optimizations such as vectorization) and on the code generated by the compiler.

\section{B. Watermark Extraction}

We present the energy, power, and execution time analysis of watermark extraction in Table III.

Again, we observe that Bruyndonckx's [26] and Koch's [31] watermarking algorithms consume much less power (up to two orders of magnitude) than others. In the case of Bruyndonckx's extraction procedure, as stated in the previous section, this is due to the locality of the embedded watermark. Also, the extraction process is restricted to the spatial domain, i.e., the search for the embedded watermark is performed in the blocks ${ }^{12}$ of the image. Whereas, in Koch's extraction procedure, the signature is extracted on the basis of the modified relationship of the DCT coefficients. It merely involves extracting the middle frequency coefficient of an $8 \times 8$ block. Thus, Koch's algorithm also requires little energy and executes fast.

\footnotetext{
${ }^{12} \mathrm{~A}$ block in an image corresponds to an $8 \times 8$ block used in discrete-cosinetransform (DCT).
}

TABLE III

WATERMARK EXTRACTION: ENERgy, POWER, AND EXECUTION TIME ANALYSIS

\begin{tabular}{c|c|c|c}
\hline Algorithm & $\begin{array}{c}\text { Energy } \\
(\mathrm{J})\end{array}$ & $\begin{array}{c}\text { Avg. Power } \\
(\mathrm{W}=\mathrm{J} / \mathrm{s})\end{array}$ & Exec.time (s) \\
\hline \hline Bruyndonckx & 0.22 & 0.79 & 0.28 \\
\hline Corvi & 70.3 & 0.47 & 150.77 \\
\hline Cox & 121 & 0.95 & 128.02 \\
\hline Dugad & 38.4 & 0.49 & 79.0 \\
\hline Fridrich & 191 & 1.1 & 173.6 \\
\hline Kim & 91.3 & 0.55 & 166.57 \\
\hline Koch & 0.61 & 0.61 & 1.0 \\
\hline Wang & 88 & 0.59 & 147.9 \\
\hline Xia & 82.7 & 0.57 & 144.51 \\
\hline Xie & 74.06 & 1.0 & 73.88 \\
\hline Zhu & 158.8 & 1.16 & 137.38 \\
\hline
\end{tabular}

On the other hand, the watermark extraction in Corvi [27] and Kim [30] is done in the wavelet domain. In this type of watermarking algorithms, we first have to determine the coefficients by calculating the wavelet transform and evaluate the decomposition levels and their sub-bands. Then, these sub-bands are used for watermark extraction. This involves complex computations and, hence, higher energy usage. Of all the watermarking algorithms presented in Table III, Fridrich [29] has the highest energy consumption and execution time because it calculates the mean and the variance of all the pixel values in the image and performs bandwise correlation and image normalization.

We find that in some cases, watermark extraction is more expensive than watermark embedding. Wavelet-transform-based watermarking techniques such as Fridrich's, compute the transform once on the input image while embedding the watermark [29]. The coefficients are first normalized (this requires mean and standard derivation calculation) and then embedded imperceptibly in the medium frequency range. On the other hand, during extraction, the transform is carried out on both the input image and the output image, and the corresponding coefficients are normalized. The correlation between the normalized coefficients of the input and output is used as a measure of the fidelity of the watermarked image. The overhead of computing 
TABLE IV

WATERMARK AUTHENTICATION: ENERGY, POWER, AND EXECUTION TIME (ONLY ON THE PDA) ANALYSIS. EXECUTION TIME ON THE PROXY SERVER IS TOO SMALL TO REPORT

\begin{tabular}{c|c|c|c}
\hline Algorithm & $\begin{array}{c}\text { Energy } \\
(\mathrm{J})\end{array}$ & $\begin{array}{c}\text { Avg. Power } \\
(\mathrm{W}=\mathrm{J} / \mathrm{s})\end{array}$ & Exec. time (s) \\
\hline \hline Bruyndonckx & 0.02 & 0.59 & 0.034 \\
\hline Corvi & 0.10 & 0.73 & 0.138 \\
\hline Cox & 0.05 & 1.35 & 0.037 \\
\hline Dugad & 0.03 & 0.97 & 0.031 \\
\hline Fridrich & 0.18 & 1.36 & 0.132 \\
\hline Kim & 0.10 & 0.76 & 0.131 \\
\hline Koch & 0.04 & 1.25 & 0.032 \\
\hline Wang & 0.08 & 1.36 & 0.059 \\
\hline Xia & 0.08 & 1.4 & 0.057 \\
\hline Xie & 0.04 & 1.0 & 0.039 \\
\hline Zhu & 0.06 & 1.2 & 0.05 \\
\hline & & &
\end{tabular}

bandwise correlation and image normalization accounts for the higher energy consumption.

\section{Watermark Authentication}

In Table IV, we list the energy, power, and execution time for watermark authentication. This task is computationally inexpensive, since it involves a simple comparison of the extracted watermark and the original watermark. From Table IV, we find that blind watermarking techniques such as Bruyndonckx's [26], in which the mark is verified without using the original image, are more power efficient as they forgo fidelity checks on the extracted image. On the other hand, fragile watermarking techniques such as Fridrich's [29], in which watermarks are designed to be distorted or "broken" under the slightest changes to the image, employ correlation measures between the original image and extracted image for image verification.

\section{IMPACT OF VARYING SECURITY AND IMAGE QUALITY ON ENERGY CONSUMPTION}

In this section, we analyze the impact of different security levels and image size/resolution on the energy consumption of the watermarking process.

\section{A. Impact of Different Security Levels}

Intuitively, it is obvious that the more secure our watermarking scheme, the more computation it will require and the more energy it will consume. In this section, we quantify this intuition by varying the security level for the watermarking algorithms used in our experiments.

In Fig. 7(a) and (b), we present the impact of increasing the security level, i.e., the number of bits used to generate the watermark (in other words, the length of the watermark), on execution time and energy requirements of watermark embedding and watermark extraction for the 11 watermarking algorithms. ${ }^{13}$ In the rest of the experiments in this paper, we used a watermark length of 32 bytes. For nearly all the watermarking algorithms,

\footnotetext{
${ }^{13}$ We say that the number of bits in a watermark is representative of the security level as more bits in a watermark would require more computational effort to extract the watermark. Recall that security is directly correlated to the computational complexity.
}

we find that the energy consumption increases almost linearly as the watermark length is increased.

From Fig. 7(a) and (b), we find that increasing the length of the watermark has little performance and energy penalty on local watermark embedding techniques, such as Bruyndonckx's [26]. Similarly, Koch's extraction process incurs minimum energy overhead with higher security levels (see discussion in previous three sections). However global embedding techniques such as Fridrich's [29], where the signature is distributed across a large number of blocks, incur significant penalty as the length of the signature is increased.

Xie [34] and Zhu's [35] algorithms embed watermarks in the frequency domain. Increased watermark length increases the computation of the correlation between frequency coefficients of the original image and the watermarked image. This correlation computation is a very complex operation and, thus, places a heavy power burden on the handheld.

This study of different security levels is useful because frequently the choice of security level must be made in conjunction with the application requirements such as real-time constraints, quality-of-service (QoS), and residual battery energy in the handheld device.

\section{B. Impact of Image Resolution and Size}

In the last set of experiments, we vary the resolution and size of the image being watermarked and study its effects on energy and execution time. The results for varying the image resolution on watermark embedding and extraction are presented in Fig. 8(a) and (b). From these figures, we observe that Fridrich's algorithm suffers maximum performance and energy penalty with increase in image resolution. As the image resolution increases, from a black and white (2-bit) image to gray-scale (8-bit) image to a color (24-bit) image, ${ }^{14}$ the number of frequency coefficients per pixel increase, even though the total number of pixels remain the same. The larger number of coefficients lead to more computations (calculation of mean, bandwise correlation, and image normalization). Thus, spatial domain filters are "immune" to image resolution variance, whereas the performance of frequency domain filters are susceptible to image resolution.

High-resolution images have higher quality, but might require more energy to download (communication energy) and to render (computational energy). On the other hand, from a security viewpoint, high resolution images provide a larger spectrum for nonuniform embedding of the watermark along each plane. ${ }^{15}$ However, this too leads to higher energy consumption, since the search space for embedding and extracting the watermark increases.

The effects of increasing image size on execution time and energy are presented in Fig. 8(c) and (d). Again, we find that higher execution time and higher energy are required for larger images. The increase is nonlinear; this is because larger images imply that there are more blocks in the image to embed the watermark in and, consequently, a larger search space for extracting and authenticating the watermark. Also, the sub-band calculation for

\footnotetext{
${ }^{14}$ Each color component RGB is assigned 8 bits.

${ }^{15}$ Each RGB component in an image forms a plane in the image.
} 

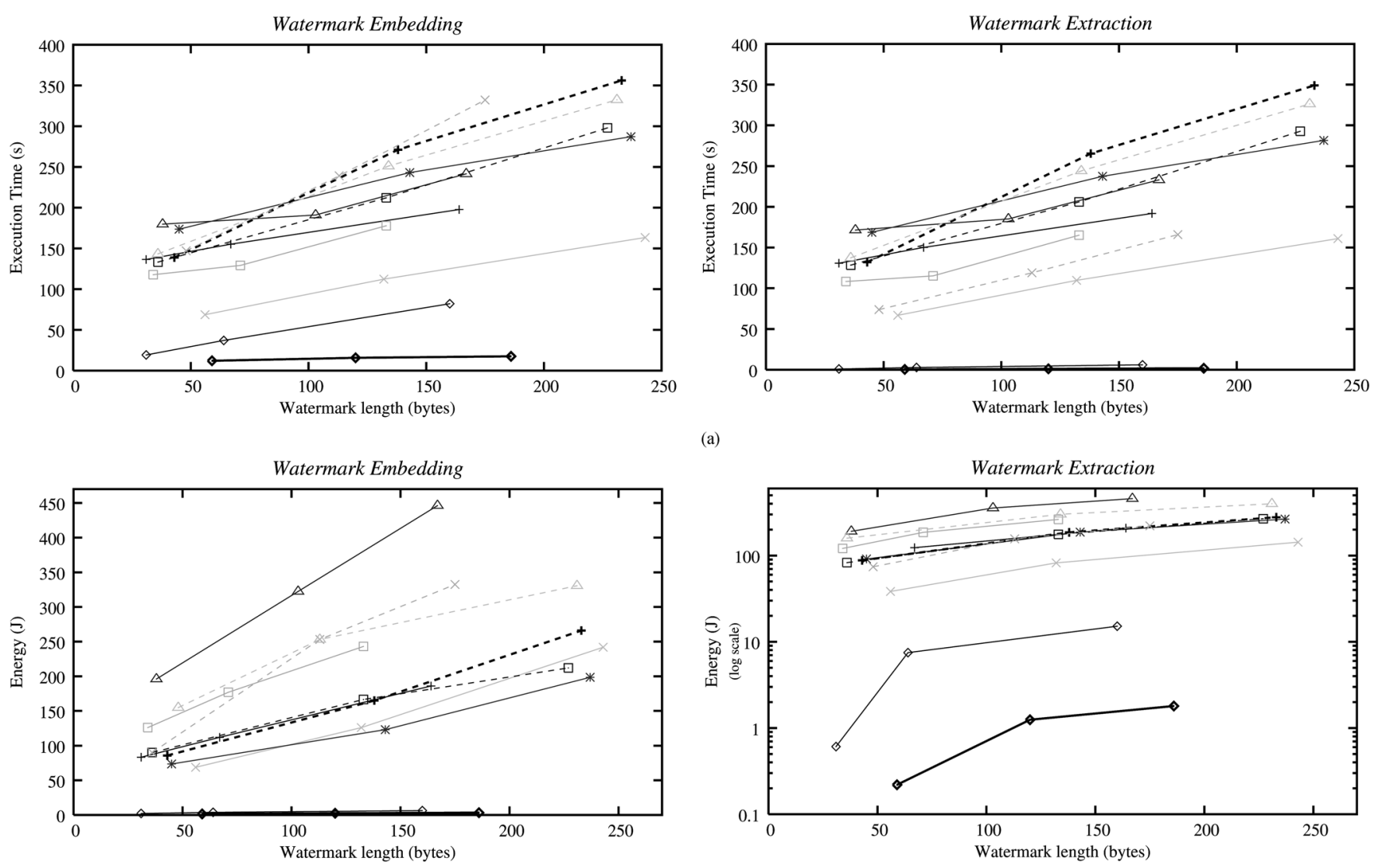

(a)

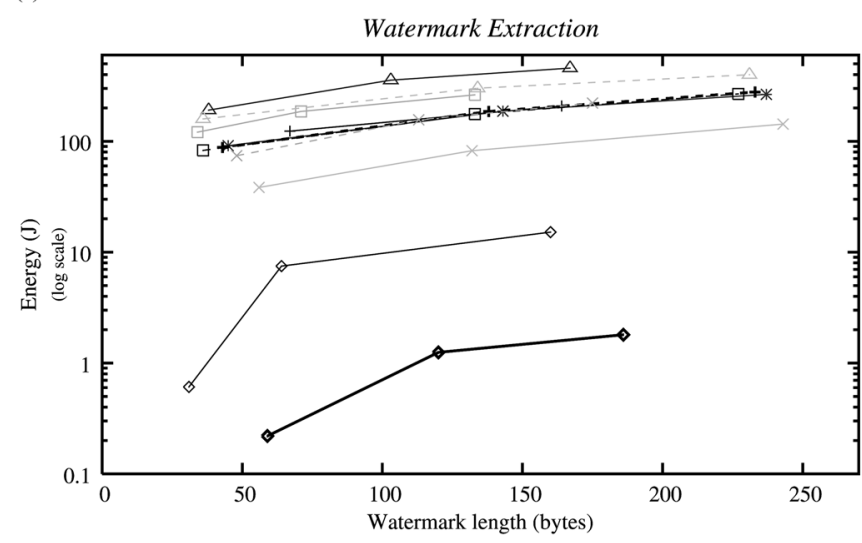

(b)

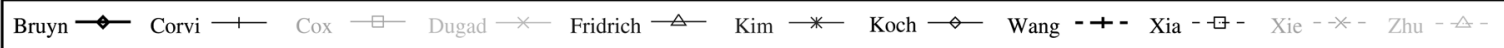

Fig. 7. Effects of increasing watermark security (length) on execution time and energy consumption. (a) Watermark length versus execution time. (b) Watermark length versus energy consumption.

each decomposition level is done within multilevel nested loops. Thus, increasing the size of the image linearly leads to higher order growth of execution time.

\section{Energy-SeCURITY TRADE-OFF IN PARTITIONING OF WATERMARKING ALGORITHMS}

In this section, we present results corresponding to the partitioning schemes discussed in Section VI-A and VI-B.

\section{A. Mapping All Watermarking Tasks to Proxy}

Table $\mathrm{V}$ lists the execution time for watermark embedding and extraction for the algorithms listed in Table I. Columns 2 and 3 correspond to the execution time of the watermark embedding process (for a gray-level image of size $512 \times 512$ ) on the proxy and the PDA, respectively, and columns 5 and 6 for the watermark extraction process. Columns 4 and 7 list the energy gain or savings achieved by "offloading" watermark embedding and extraction to the proxy. This is in effect the energy required to execute the entire watermarking embedding and extraction algorithms on the handheld. We find that energy savings vary from $47 \mathrm{~J}$ to a high of $236 \mathrm{~J}$.

We also note from the execution time results that migrating the entire watermarking procedure to the proxy also boosts performance by over two orders of magnitude. Thus, the dual performance and energy benefit makes this partitioning highly at- tractive for environments where the handheld communicates with a trusted proxy over a secure channel such as SSL.

\section{B. Security-Driven Partitioning}

We partitioned the watermarking algorithms as discussed in Section VI-B and executed them using our proxy server along with the Zaurus PDA. The execution time results for the tasks run on the proxy and the PDA for watermark embedding and extraction are listed in Table VI.

The results in this table demonstrate that our security-driven task partitioning leads to very low execution times as compared to executing the watermarking on the PDA (as listed earlier in Table V). In fact, the total execution time is comparable to executing watermarking exclusively on the proxy (compare with Table V).

We present the energy savings from the security-driven partitioning over executing watermarking on the handheld in Fig. 9. Clearly, we achieve large savings, over $80 \%$, for all the watermarking algorithms using our partitioning scheme. Note that in absolute numbers the watermarking tasks run on the PDA consume about $30-50 \mathrm{~J}$.

\section{Communication Overhead}

The communication overheads due to the security-driven partitioning scheme are shown diagrammatically in Fig. 10. During embedding, the PDA sends the image and watermark to the 

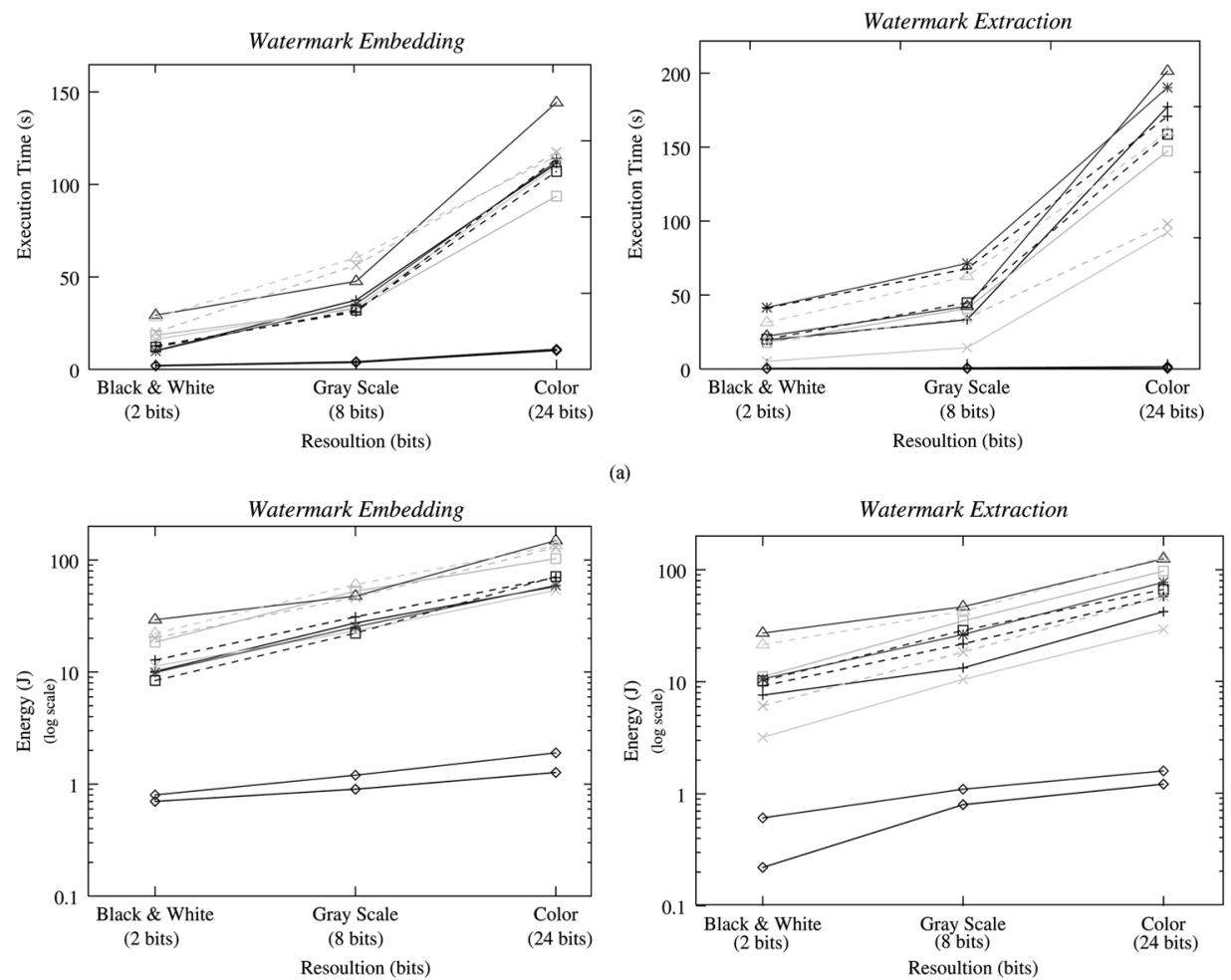

(b)
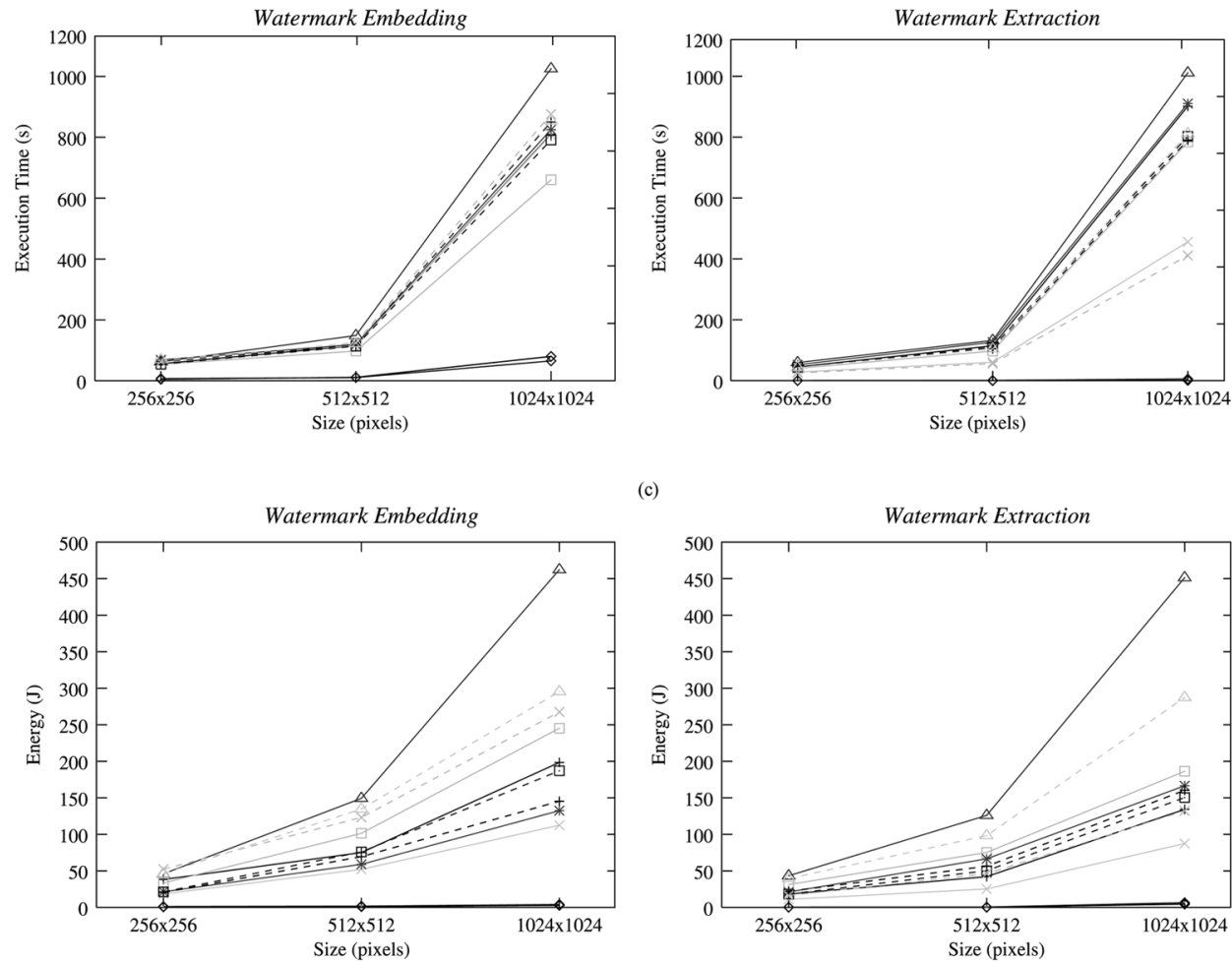

(d)

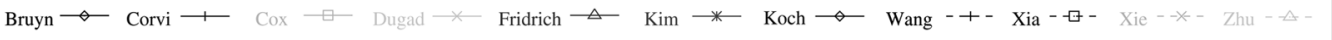

Fig. 8. Effects of increasing image resolution and size on execution time and energy consumption. (a) Impact of image resolution on execution time. (b) Impact of image resolution on energy. (c) Impact of image size on execution time. (d) Impact of image size on energy consumption.

proxy for preprocessing. The proxy sends the coefficients back to the PDA. During extraction, the PDA sends the decrypted coefficients to the proxy, who then sends back the extracted watermark. Among these, the only significant communication over- heads are due to the transfer of the image and watermark between the PDA and the proxy. We list the transfer times and communication energy associated with these transfers during watermark embedding and extraction in Table VII. The results 
TABLE V

EXECUTION TIME AND ENERGY ANALYSIS WHEN ALL THE WATERMARKINC TASKS ARE OFFLOADED TO THE PROXY Versus WHEN THEY ARE RUN ON THE PDA

\begin{tabular}{c|c|c|c|c|c|c}
\hline & \multicolumn{3}{|c|}{ Embedding } & \multicolumn{3}{c}{ Extraction } \\
Algo. & Exec. time(s) & Energy & \multicolumn{2}{c}{ Exec. time(s) } & Energy \\
& Pro xy & PD A & Gain (J) & Pro xy & PD A & Gain (J) \\
\hline \hline Corvi & 0.79 & 211.58 & 129.29 & 0.74 & 213.96 & 99.76 \\
\hline Dugad & 0.75 & 212.50 & 106.84 & 0.34 & 98.10 & 47.68 \\
\hline Kim & 0.83 & 217.73 & 113.65 & 0.79 & 200.92 & 110.13 \\
\hline Wang & 0.84 & 217.13 & 132.88 & 0.74 & 198.70 & 118.23 \\
\hline Xia & 0.85 & 202.08 & 135.90 & 0.78 & 191.46 & 109.57 \\
\hline Xie & 0.77 & 211.87 & 223.01 & 0.38 & 97.02 & 92.26 \\
\hline Zhu & 0.82 & 222.59 & 252.88 & 0.71 & 204.45 & 236.33 \\
\hline
\end{tabular}

TABLE VI

EXECUTION TIMES WHEN THE WATERMARKING TASKS ARE Partitioned BetWEEN THE PROXY AND THE HANDHELD Without COMPROMISING SECURITY

\begin{tabular}{c|c|c|c|c|c|c}
\hline \multirow{2}{*}{ Algorithm } & \multicolumn{3}{|c|}{ Embedding } & \multicolumn{3}{c}{ Extraction } \\
& \multicolumn{2}{c}{ Exec. Time(s) } & \multicolumn{2}{c}{ Exec. Time(s) } \\
& Proxy & PDA & Total & Proxy & PDA & Total \\
\hline \hline Corvi & 0.69 & 0.36 & 1.05 & 0.63 & 0.41 & 1.04 \\
\hline Dugad & 0.68 & 0.19 & 0.87 & 0.31 & 0.15 & 0.46 \\
\hline Kim & 0.70 & 0.53 & 1.23 & 0.65 & 0.48 & 1.13 \\
\hline Wang & 0.69 & 0.56 & 1.25 & 0.67 & 0.64 & 1.31 \\
\hline Xia & 0.69 & 0.46 & 1.15 & 0.61 & 0.55 & 1.16 \\
\hline Xie & 0.68 & 0.41 & 1.09 & 0.31 & 0.18 & 0.49 \\
\hline Zhu & 0.70 & 0.65 & 1.35 & 0.64 & 0.64 & 1.28 \\
\hline & & & & & &
\end{tabular}

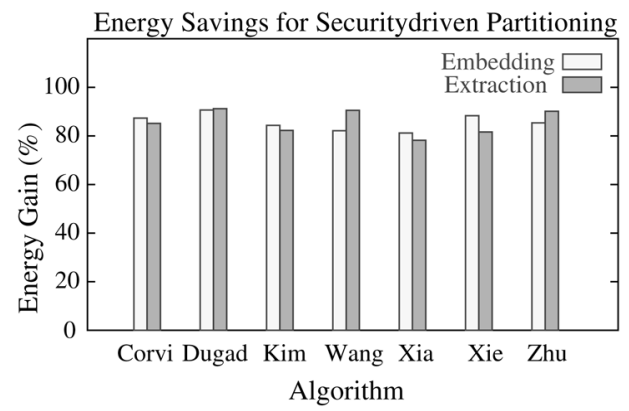

Fig. 9. Energy savings for the security-driven partitioning scheme over executing the entire watermarking algorithm on the PDA.

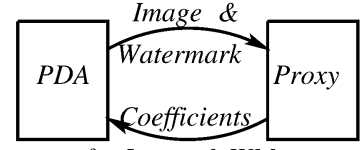

for Image \& $W M$

(a)

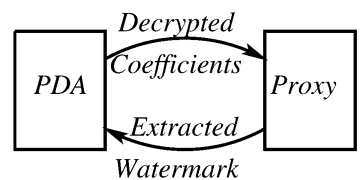

(b)
Fig. 10. Communication overheads of: (a) watermark embedding and (b) watermark extraction.

presented in Table VII were measured not estimated; also, these include the SSL channel effects. The overheads are very small compared to the watermarking tasks that run on the PDA $(0.4 \mathrm{~J}$ for transfer versus about 30-50 J for the watermarking process). The coefficients of the image and the watermark are smaller than
TABLE VII

Time AND ENERgy Overheads DuE to COMMUNiCATION BETweEn ProXy AND PDA IN THE SECURITY-DRIVEN PARTITIONING SCHEME

\begin{tabular}{|c|c|c|c|c|}
\hline \multirow[b]{2}{*}{ Algo } & \multicolumn{2}{|c|}{ Embedding } & \multicolumn{2}{|l|}{ Extraction } \\
\hline & $\begin{array}{l}\text { Image Transf. } \\
\text { Time }(\mathrm{sec})\end{array}$ & $\begin{array}{l}\text { Assoc. } \\
\text { Energy }\end{array}$ & $\begin{array}{c}\text { Watermark Transf. } \\
\text { Time }(\mathrm{sec})\end{array}$ & $\begin{array}{l}\text { Assoc. } \\
\text { Energy }\end{array}$ \\
\hline Corvi & 0.73 & $400 \mathrm{~mJ}$ & 0.11 & $55 \mathrm{~mJ}$ \\
\hline Dugad & 0.70 & $393 \mathrm{~mJ}$ & 0.12 & $57 \mathrm{~mJ}$ \\
\hline Kim & 0.74 & $405 \mathrm{~mJ}$ & 0.10 & $51 \mathrm{~mJ}$ \\
\hline Wang & 0.75 & $410 \mathrm{~mJ}$ & 0.12 & $58 \mathrm{~mJ}$ \\
\hline $\mathrm{Xia}$ & 0.69 & $387 \mathrm{~mJ}$ & 0.12 & $58 \mathrm{~mJ}$ \\
\hline Xie & 0.76 & $415 \mathrm{~mJ}$ & 0.10 & $52 \mathrm{~mJ}$ \\
\hline Zhu & 0.73 & $401 \mathrm{~mJ}$ & 0.10 & $50 \mathrm{~mJ}$ \\
\hline
\end{tabular}

half the size of the image and watermark; hence, they add only a small energy overhead to the partitioned process.

\section{CONClusion}

We presented energy characterization of several waveletbased image watermarking algorithms. We proposed two proxy-based partitioning techniques for energy efficient watermarking on mobile devices. These techniques partition watermarking tasks between a proxy server and the handheld device. Energy consumption due to watermarking tasks can be minimized for the handheld by offloading these tasks completely to the proxy server at the cost of security. Our approach seeks to maximize energy savings while ensuring secure access to media data. We demonstrated through experimental results and energy measurements of several wavelet-based image watermarking algorithms on a PDA that our security-driven partitioning scheme leads to an average $80 \%$ reduction in the energy, while achieving over two orders of magnitude improvement in performance over executing watermarking on the handheld. Clearly, no approach that relies on network communication and/or another computer for offloading computation can be $100 \%$ secure. However, we believe that our partitioning scheme is a balance between reducing energy and securing the data. In future work, we plan to study energy and security-aware partitioning scheme for video watermarking.

\section{ACKNOWLEDGMENT}

The first author, A. Kejariwal, would like to thank P. Meerwald for providing the source code of the watermarking algorithms. He would also like to thank R. Cornea, M. Luthra, and N. Bansal for their valuable suggestions for the preparation of the paper published in the Design Automation Conference, June 2004.

\section{REFERENCES}

[1] K. Tanaka, Y. Nakamura, and K. Matsui, "Embedding secret information into a dithered multilevel image," in Proc. IEEE Military Commun. Conf., 1990, pp. 216-220.

[2] F. Pérez-González and J. R. Hernández, "A tutorial on digital watermarking," in Proc. IEEE Annu. Carnahan Conf. Security Technol., 1999, pp. 286-292.

[3] L. Qiao, Multimedia Security and Copyright Protection Dept. Comput. Sci., Univ. Illinois, Urbana-Champaign. Urbana-Champaign, IL, 1998, Ph.D. dissertation. 
[4] O. Esparza, M. Fernandez, and M. Soriano, "Protecting mobile agents by using traceability techniques," in Proc. Int. Conf. Inform. Technol., 2003, pp. 264-268.

[5] D. V. Thanh, "Security issues in mobile ecommerce," in Proc. Int. Workshop Database Expert Syst. Appl., 2000, pp. 412-425.

[6] P. Kocher, R. Lee, G. McGraw, A. Raghunathan, and S. Ravi, "Security challenges in embedded system design," in Proc. 41st Des. Automat. Conf., 2004, pp. 753-760.

[7] W. Dietl, P. Meerwald, and A. Uhl, "Protection of wavelet-based watermarking systems using filter parametrization," IEEE Trans. Signal Process., vol. 83, no. 10, pp. 2095-2116, Oct. 2003.

[8] H. C. Rao, D. Chang, Y. Chen, and M. Chen, "iMobile: A proxy-based platform for mobile services," in Proc. Wireless Mobile Internet, 2001, pp. 3-10.

[9] J. G. Steiner, B. C. Neumann, and J. I. Schiller, "Kerberos: An authentication service for open network systems," in Proc. Winter USENIX Conf., 1988, pp. 191-201.

[10] B. C. Neuman, "Proxy-based authorization and accounting for distributed systems," in Proc. Int. Conf. Distributed Computing Syst., 1993, pp. 283-291.

[11] A. Fox and S. D. Gribble, "Security on the move: Indirect authentication using kerberos," in Proc. Mobile Computing Networking, White Plains, NY, 1996, pp. 155-164.

[12] B. Zenel, A Proxy Based Filtering Mechanism for the Mobile Environment Comput. Sci. Dept., Columbia University, New York, 1995, Tech. Rep. CUCS-0-95.

[13] M. Burnside, D. Clarke, T. Mills, A. Maywah, S. Devadas, and R. Rivest, "Proxy-based security protocols in networked mobile devices," in Proc. ACM Symp. Appl. Comput., 2002, pp. 265-272.

[14] N. R. Potlapally, S. Ravi, A. Raghunathan, and N. K. Jha, "Analyzing the energy consumption of security protocols," in Proc. Int. Symp. Low Power Electron. Des., 2003, pp. 30-35.

[15] A. Rudenko, P. Reiher, G. J. Popek, and G. H. Kuenning, "The remote processing framework for portable computer power saving," in Proc. 1999 ACM Symp. Appl. Comput., 1999, pp. 365-372.

[16] U. Kremer, J. Hicks, and J. Rehg, Compiler-directed remote task execution for power management: A case study, Compaq Cambridge Research Laboratory (CRL), Cambridge, MA, 2000, Tech. Rep. 2000-2.

[17] P. Rong and M. Pedram, "Extending the lifetime of a network of battery-powered mobile devices by remote processing: A markovian decision-based approach," in Proc. 40th Conf. Des. Automat., 2003, pp. 906-911.

[18] G. Voyatzis and I. Pitas, "Embedding robust logo watermarks in digital images," Proc. Digital Signal Process., pp. 213-216, 1987.

[19] C. Hsu and J. Wu, "Hidden signatures in images," in Proc. IEEE Int. Conf. Image Process., 1996, pp. 223-226.

[20] R. Dugad, K. Ratakonda, and N. Ahuja, "A new wavelet-based scheme for watermarking images," in Proc. Int. Conf. Image Process., 1998, pp. $357-372$.

[21] T. Kalker, G. Depovere, J. Haitsma, and M. Maes, "A video watermarking system for broadcast monitoring," in Proc. SPIE Electron. Imaging, Security Watermarking Multimedia Contents, 1999, pp. 103-112.

[22] H. Inoue, A. Miyazaki, A. Yamamoto, and T. Katsura, "A digital watermark based on the wavelet transform and its robustness on image compression," in Proc. IEEE Int. Conf. Image Process., 1998, pp. 391-395.

[23] F. Hartung, J. K. Su, and B. Girod, "Spread spectrum watermarking: Malicious attacks and counterattacks," in Security Watermarking Multimedia Contents, 1999, pp. 147-158.

[24] I. Daubechies, "Orthonormal bases of compactly supported wavelets," Comm. Pure Appl. Math., pp. 909-996, 198.

[25] W. Diffie and M. E. Hellman, "New directions in cryptography," IEEE Trans. Inform. Theory, vol. IT-22, no. 6, pp. 644-654, Nov. 1976.

[26] O. Bruyndonckx, J.-J. Quisquater, and B. Macq, "Spatial method for copyright labeling of digital images," in Proc. IEEE Workshop Nonlinear Signal Image Process., 1995, pp. 456-459.

[27] M. Corvi and G. Nicchiotti, "Wavelet-based image watermarking for copyright protection," in Proc. Scandinavian Conf. Image Anal., 1997, pp. 157-163.

[28] I. Cox, J. Kilian, T. Leighton, and T. Shamoon, "Secure spread spectrum watermarking for multimedia," IEEE Trans. Image Process., vol. 6, no. 12, pp. 1673-1687, Dec. 1997.

[29] J. Fridrich, "Combining low-frequency and spread spectrum watermarking," in Proc. Int. Symp. Optical Sci. Eng. Instrumentation, 1998, pp. $19-24$.
[30] J. R. Kim and Y. S. Moon, "A robust wavelet-based digital watermark using level-adaptive thresholding," in Proc. Int. Conf. Image Process., 1999, pp. 226-230.

[31] E. Koch and J. Zhao, "Towards robust and hidden image copyright labeling," in Proc. Int. Workshop Nonlinear Signal Image Process., 1995 pp. $452-455$.

[32] H. M. Wang, P. Su, and C. J. Kuo, "Wavelet-based digital image watermarking," Opt. Expr., vol. 3, no. 12, pp. 491-496, Dec. 1998.

[33] X. G. Xia, C. G. Boncelet, and G. R. Arce, "Wvelet transform based watermarking for digital images," Opt. Expr., vol. 3, no. 1, pp. 497-511, Dec. 1998 .

[34] L. Xie and G. Arce, "Joint wavelet compression and authentication watermarking," in Proc. Int. Conf. Image Process., 1998, pp. 427-431.

[35] W. Zhu, Z. Xiong, and Y. Q. Zhang, "Multiresolution watermarking for images and video: A unified approach," in Proc. Int. Conf. Image Process., 1998, pp. 465-468.

[36] P. Meerwald, "Digital Image Watermarking in the Wavelet Transform Domain," M.S. thesis, Dept. Scientific Computing, Univ. Salzburg, Salzburg, Austria, 2001.

[37] S. Voloshynovskiy, S. Pereira, and T. Pun, "Watermark attacks," in Proc. Erlangen Watermarking Workshop, 1999.

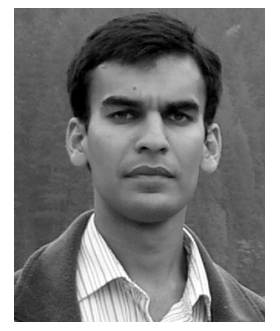

Arun Kejariwal (S'02) received the B. Tech. degree in electrical engineering from the Indian Institute of Technology (IIT), New Delhi, India, in 2002.

$\mathrm{He}$ is currently a fourth year doctoral student at the Center for Embedded Computer Systems, University of California, Irvine. His interests include parallelizing and optimizing compilers, multithreaded and multicore architectures, and low-power embedded systems. He served as the Student Editor of IEEE Potentials from 2004 to 2006.

Mr. Kejariwal was a recipient of the Best Student Paper Award at the 6th International Symposium on High Performance Computing (ISHPC-VI) in 2005.

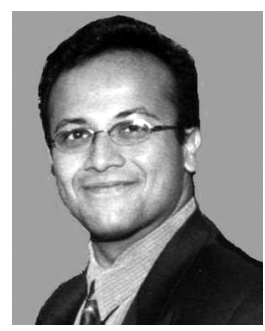

Sumit Gupta (M’03) received the Ph.D. degree from the University of California, Irvine, in 2003 , and the B.Tech. degree from the Indian Institute of Technology (IIT), New Delhi, India, in 1995.

For his Ph.D., he developed a novel parallelizing approach to the high-level synthesis of hardware from behavioral $\mathrm{C}$ that blended parallelizing compiler techniques with traditional high-level synthesis techniques. The software framework, called SPARK, that resulted from this work has been downloaded by almost 10,000 users and has been licensed by an EDA startup for commercialization. He has since worked on reconfigurable systems and field-programmable gate arrays (FPGAs). He is currently at Tensilica Inc., Santa Clara, CA, where he is in charge of product definition and future roadmap of the company's configurable processor cores.

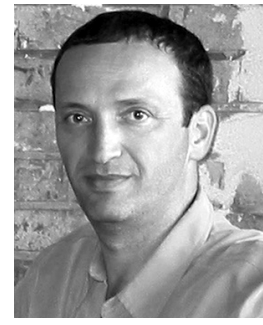

Alexandru Nicolau (M'85) received the Ph.D. degree in computer science from Yale University, New Haven, CT, in 1984.

He was an Assistant Professor at Cornell University, Ithaca, NY, until 1988, when he joined the University of California, Irvine, where he is currently a Professor of Computer Science and Electrical Engineering and Computer Science. His research interests include embedded systems, parallel computing, parallelizing compilers, computer architecture, and optimizing compilers. He has authored over 200 scientific papers and several books.

Dr. Nicolau currently serves as Editor-in-Chief of the International Journal of Parallel Programming. He has served on the steering and program committees of several conferences and workshops, including ICS, MICRO, PACT, and LCPC. 


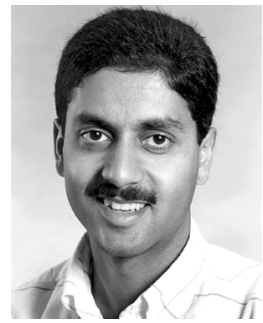

Nikil D. Dutt (SM'96) received the Ph.D. in computer science from the University of Illinois at Urbana-Champaign, Urbana-Champaign, in 1989.

$\mathrm{He}$ is currently a Professor of Computer Science and Electrical Engineering and Computer Science at the University of California (UCI), and is affiliated with the following centers at UCI: CECS, CPCC, and CAL-IT2. His research interests include embedded systems design automation, computer architecture, optimizing compilers, system specification techniques, and distributed embedded systems. He currently serves as Editor-in-Chief of ACM Transactions on Design Automation of Electronic Systems (TODAES) and as Associate Editor of ACM Transactions on Embedded Computer Systems (TECS). He was an ACM SIGDA Distinguished Lecturer during 2001-2002 and an IEEE Computer Society Distinguished Visitor from 2003 to 2005 . He has served on the steering, organizing, and program committees of several premier Computer-Aided Design (CAD) and Embedded System Design conferences and workshops, including ASPDAC, CASES, CODES+ISSS, DATE, ICCAD, ISLPED and LCTES. He serves or has served on the advisory boards of ACM SIGBED and ACM SIGDA, and is Vice-Chair of IFIP WG 10.5.

He was a recipient of the Best Paper Awards at CHDL89, CHDL91, VLSIDesign 2003, CODES+ISSS 2003, and ASPDAC-2006.

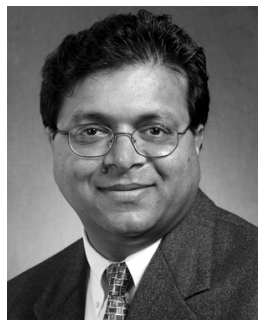

Rajesh Gupta (F'04) received the B. Tech. degree in electrical engineering from the Indian Institute of Technology (IIT), Kanpur, India, in 1984, the M.S. degree in electronic engineering and computer science from the University of California, Berkeley, in 1986, and the Ph.D. in electrical engineering from Stanford University, Stanford, CA, in 1994.

$\mathrm{He}$ is currently a Professor of Computer Science and holder of the QUALCOMM Endowed Chair in Embedded Microsystems at the University of California, San Diego. His current research is focused on energy efficient and mobile computing issues in embedded systems. He is author/co-author of over 150 articles on various aspects of embedded systems and design automation, and holds four patents on PLL design, data-path synthesis, and system-on-chip (SOC) modeling.

Dr. Gupta served as founding Chair of the ACM/IEEE Conference on Models and Methods in Codesign (MEMOCODE) and founding Co-Chair of ACM/ IEEE/IFIP Conference on Codesign and System Synthesis (CODES+ISSS). He is Editor-in-Chief Emeritus of the IEEE DESIGN \& TEST OF COMPUTERS and serves on the editorial boards of the IEEE TRANSACTIONS ON COMPUTER-AIDED DESIGN OF INTEGRATED CIRCUITS AND SYSTEMS, and chairs the Steering Committee of IEEE TRANSACTIONS ON MOBILE COMPUTING. He serves as the Vice President of Publications of the IEEE Council on Electronic Design Automation (CEDA). He is a distinguished lecturer for the ACM/SIGDA and the IEEE Circuits and Systems (CAS) Society. He is a recipient of the Chancellor's Fellow at the University of California at Irvine, UCI Chancellor's Award for excellence in undergraduate research, National Science Foundation CAREER Award, two Departmental Achievement Awards, and a Components Research Team Award at Intel. 